\title{
Air in the L4-5 Epidural Space Appearing as Disc Herniation
}

\author{
Angud S. Mehdi, BA; Peter S. Amenta, MD; James S. Harrop, MD
}

Neurosurgery Department, Thomas Jefferson University Hospitals, Philadelphia, PA

\section{ABSTRACT}

Disc degeneration or spondylosis, when severe, may lead to the development of a vacuum phenomenon in the spine caused by gas production. This gas is visible on plain X-ray films, and is even more clearly apparent on computed tomography (CT) imaging, but may not be apparent on MRI. We present the case of a 69 year-old female with MRI appearing to exhibit a significant L4-5 disc herniation that, on further imaging with CT, was determined to be air in the epidural space without disc herniation. The importance of correlating clinical presentation, neurologic examination, and multimodal imaging is stressed.

\section{CASE REPORT}

History and Presentation: A 69-year old female presented with musculoskeletal low back pain extending into her hips and thighs, in a non-radicular manner. She was neurologically intact and had received physical therapy and epidural steroid injections without symptomatic relief.

Imaging: Anterior-posterior and lateral plain films of the thoracic and lumbar spine were unremarkable. T2-weighted MRI images showed marked loss of disc height at L4-L5, with a large central disc herniation and an "extruded disc fragment" resulting in left greater than right foraminal stenosis (Figures 1). A subsequent spine CT, however, revealed air in the L4-L5 disc space consistent with vacuum disc phenomenon which was chronic and appeared on abdominal ct scans done several years prior (Figure 2).

\section{DISCUSSION}

Gas production or "vacuum phenomenon" in the intervertebral space may be a byproduct of disc degeneration. Accumulated gas is composed of nitrogen and carbon dioxide and remains within the disc space. ${ }^{1,2}$ Gas may escape into the epidural space through a fissure in the annulus fibrosus. The vast majority of individuals with epidural gas are asymptomatic.

Gas in the intervertebral disc space is a relatively common radiologic finding and is found in approximately $46 \%$ of CT examinations. 3 Air in the disc can be seen as a signal void on T1- and T2-weighted MRI, however, CT is more sensitive for identifying air in the disc or epidural space.

On T2-weighted MRI, disc herniations appear as iso- to hypointense material extending beyond the confines of the disc space. These findings are often associated with the loss of disc height at the level of the herniated disc., ${ }^{4,5}$ These very findings were observed in the T2-weighted imaging of our patient (Figures 1 and 2).

Our patient presented with low back pain and no symptoms consistent with radiculopathy referable to L4-L5. Despite the findings seen on MRI, the CT clearly illustrated air in the L4-L5 disc space and left anterolateral portion of the epidural space (Figure 2). In light of the clinical presentation and CT findings, the MRI findings were deemed to be the result of epidural air and the patient was treated with conservative management.

We present a case of contrasting imaging findings as an example of the importance of correlating clinical presentation, neurologic examination, and multimodal imaging in the treatment of back pain.

\section{REFERENCES}

1. Ford LT, Gilula LA, Murphy WA, Gado M. Analysis of gas in vacuum lumbar disc. AJR Am J Roentgenol. Jun 1977;128(6):1056-1057.

2. Yoshida H, Shinomiya K, Nakai O, Kurosa Y, Yamaura I. Lumbar nerve root compression caused by lumbar intraspinal gas. Report of three cases. Spine (Phila Pa 1976). Feb 1 1997;22(3):348-351.

3. Larde D, Mathieu D, Frija J, Gaston A, Vasile N. Spinal vacuum phenomenon: CT diagnosis and significance. J Comput Assist Tomogr. Aug 1982;6(4):671-676.

4. Videman T, Battie MC, Gill K, Manninen H, Gibbons LE, Fisher LD. Magnetic resonance imaging findings and their relationships in the thoracic and lumbar spine. Insights into the etiopathogenesis of spinal degeneration. Spine (Phila Pa 1976). Apr 15 1995;20(8):928-935.

5. Hashimoto K, Akahori O, Kitano K, Nakajima K, Higashihara T, Kumasaka Y. Magnetic resonance imaging of lumbar disc herniation. Comparison with myelography. Spine (Phila Pa 1976). Nov 1990;15(11):1166-1169.

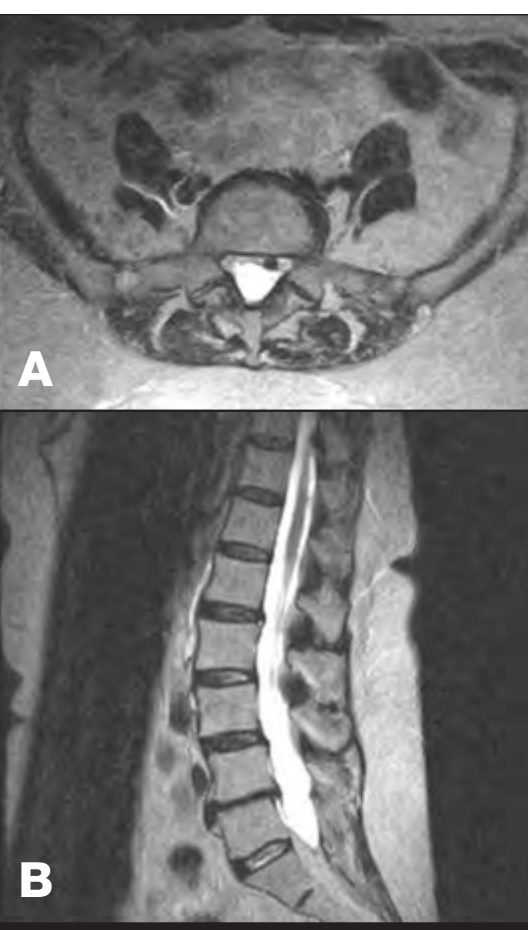

Figure 1

(A) T2-weighted axial MRI shows a hypointense area in the left anterolateral epidural space. Initially, this was initially thought to be a disc herniation and later revised upon CT evaluation to be an incidental finding of air in the epidural space. (B) T2-weighted saggital MRI.

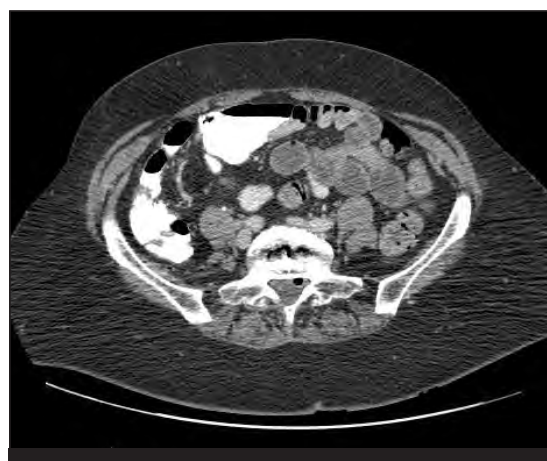

Figure 2

Axial CT image clearly shows low attenuation signal representative of air in the medial disc and left anterolateral epidural spaces. 